University of Wollongong

Research Online

Faculty of Engineering and Information

Faculty of Engineering and Information

Sciences - Papers: Part B

Sciences

2019

\title{
A 7R Spatial Linkage for Ankle Rehabilitation with an Arbitrary Ankle Rotation Axis
}

Chih-Ching Hsieh

National Taiwan University of Science and Technology

Chin-Hsing Kuo

National Taiwan University of Science and Technology, University of Wollongong, chkuo@uow.edu.au

Daisuke Matsuura

Tokyo Institute Of Technology

Yukio Takeda

Tokyo Institute Of Technology

Follow this and additional works at: https://ro.uow.edu.au/eispapers1

Part of the Engineering Commons, and the Science and Technology Studies Commons

Research Online is the open access institutional repository for the University of Wollongong. For further information contact the UOW Library: research-pubs@uow.edu.au 


\title{
A 7R Spatial Linkage for Ankle Rehabilitation with an Arbitrary Ankle Rotation Axis
}

\author{
Abstract \\ In this paper, a 7R spatial linkage for ankle rehabilitation was proposed. Thanks to its kinematic geometry, \\ the output joint, which is accommodated by the patient's ankle, of the linkage can possess an arbitrary \\ joint axis at any configuration. This advantage allows the patient achieving a flexible ankle motion in 3-D \\ space while performing the rehabilitation exercise. The design concept of the 7R linkage is explained, and \\ the displacement and force relationships between the input and output joints (i.e., the motor and patient's \\ ankle) are analyzed through an equivalent RSSR linkage. A prototype is built to validate the proposed \\ design concept. \\ Disciplines \\ Engineering | Science and Technology Studies

\section{Publication Details} \\ Hsieh, C., Kuo, C., Matsuura, D. \& Takeda, Y. (2019). A 7R Spatial Linkage for Ankle Rehabilitation with an \\ Arbitrary Ankle Rotation Axis. Mechanisms and Machine Science, 73 1491-1498. Krakow, Poland \\ Advances in Mechanism and Machine Science: Proceedings of the 15th IFToMM World Congress on \\ Mechanism and Machine Science
}




\title{
A 7R Spatial Linkage for Ankle Rehabilitation with an Arbitrary Ankle Rotation Axis
}

\author{
Chih-Ching Hsieh ${ }^{1}$, Chin-Hsing Kuo ${ }^{1,2}$, Daisuke Matsuura ${ }^{3}$, Yukio Takeda ${ }^{3}$ \\ ${ }^{1}$ National Taiwan University of Science and Technology, Taipei 106, Taiwan \\ ${ }^{2}$ University of Wollongong, NSW 2522, Australia \\ ${ }^{3}$ Tokyo Institute of Technology, Tokyo 152-8550, Japan \\ chkuo@uow.edu. au
}

\begin{abstract}
In this paper, a 7R spatial linkage for ankle rehabilitation was proposed. Thanks to its kinematic geometry, the output joint, which is accommodated by the patient's ankle, of the linkage can possess an arbitrary joint axis at any configuration. This advantage allows the patient achieving a flexible ankle motion in 3-D space while performing the rehabilitation exercise. The design concept of the 7R linkage is explained, and the displacement and force relationships between the input and output joints (i.e., the motor and patient's ankle) are analyzed through an equivalent RSSR linkage. A prototype is built to validate the proposed design concept.
\end{abstract}

Keywords: Ankle Rehabilitation, 7R Linkage, RSSR Mechanism.

\section{$1 \quad$ Introduction}

The human ankle is a complex rotational joint for which the rotation axis is changed at different ankle postures. However, most ankle rehabilitation devices were designed to produce a rotational motion with an invariant rotation axis (e.g., [1-3]) to guide the ankle performing the dorsiflexion/plantarflexion (DF/PF) motion. Such an invariantrotation-axis design may conflict with the nature of ankle motion. To deal with this problem, an ankle rehabilitation apparatus with variable ankle joint axis had been proposed by Matsuura et al. [4]. It is a special 5R2P linkage and has a passive revolute (R) joint that possesses a changeable axis for adapting the complex ankle motion. In other words, the instantaneous rotation axis of this joint is defined by the ankle motion at any instant.

Inspired by the special 5R2P linkage, this paper proposes a modified concept of such a spatial linkage, presenting a 7R spatial linkage for ankle rehabilitation. The motivation of this modification is to improve the transmission efficiency of the linkage by replacing the prismatic $(\mathrm{P})$ joints to revolute joints.

In what follows, the kinematic structure of the 5R2P linkage will be reviewed first with a particular focus on its adaptive rotational degree of freedom (DoF) for ankle motion. Accordingly, a 7R linkage is introduced, and its kinematic and force analyses 
will be carried out through an equivalent RSSR linkage. Based on the analysis results, a prototype was built up.

\section{A 5R2P Linkage for Ankle Rehabilitation Device: A Review}

In [4], a spatial 5R2P linkage with a variable ankle rotation axis was proposed for ankle rehabilitation, Fig. 1. The design concept is based on the Oldham's coupling (Fig. 1(a)), which transmits powers between two parallel rotational axes via a planar RPPR linkage. The two rotational joints are then used as the actuated motor and adapting to the patient's ankle, respectively. And, as illustrated in Fig. 1(a), the motion of the linkage is always effective wherever the equivalent rotational joint of the ankle is. Based on this concept, the planar RPPR linkage was then extended to a spatial 5R2P linkage, Fig. 1(b), for transmitting motion between two rotational axes in space. As illustrated in Fig. 1(b), the changing rotation axis of the ankle joint $\left(\mathrm{J}_{7}\right)$ can adapt to the linkage motion at any configuration.

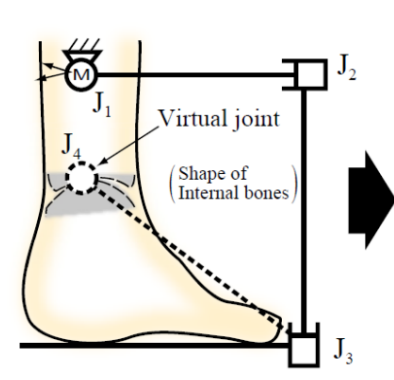

(a) Oldham's coupling (a planar RPPR linkage)

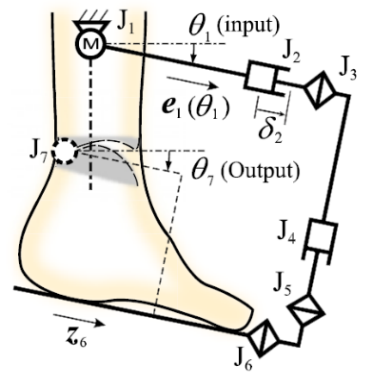

(b) A spatial 5R2P linkage

Fig. 1. A spatial 5R2P linkage that has a passive rotational joint with a variable rotational axis $\left(\mathrm{J}_{4}\right.$ in Fig. 1(a) and $\mathrm{J}_{7}$ in Fig. 1(b)) adapted to the ankle motion [4].

\section{$3 \quad$ A 7R Linkage for Ankle Rehabilitation with an Arbitrary Rotational Axis of Ankle Motion}

\subsection{The 7R Linkage}

Inspired by the 5R2P linkage shown in Fig. 1(b), a 7R linkage was devised for enhancing the transmission efficiency for ankle rehabilitation. The proposing design is shown in Fig. 2(a). The main design strategy here is to replace the two P joints of the 5R2P linkage by two $\mathrm{R}$ joints such that the structure compactness and transmission efficiency of the linkage can be inherently promoted. Besides, for design simplicity, we make joints $R_{2}, R_{3}$ and $R_{4}$ intersect at a point, while joints $R_{4}$, $R_{5}$, and $R_{6}$ do at another point. The other two remaining joints represent the actuation and the passive ankle joint, respectively, as denoted on Fig. 2(a). Based on this arrangement, the ankle 
joint can be driven by the actuated joint at an arbitrary ankle joint position and orientation. That is, for the shown one-degree-of-freedom 7R linkage, its mobility is independent of the position and orientation of the ankle joint. In this way, the rehabilitative ankle motion can be motivated with an arbitrary rotational axis that is accommodated with patient's anatomy of the ankle at any instant.

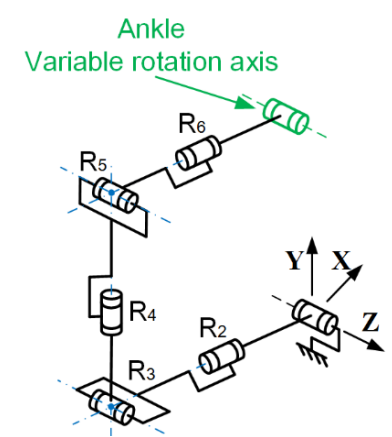

(a) The 7R linkage

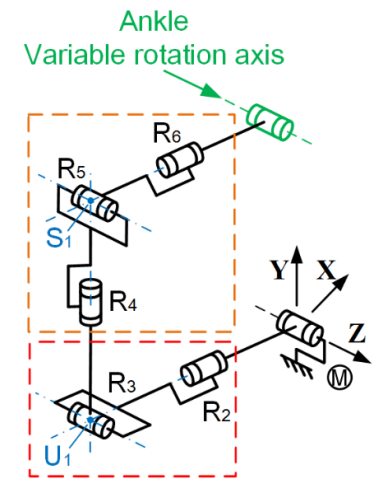

(b) The equivalent RUSR linkage

Fig. 2. A closed-loop 7R linkage for ankle rehabilitation and its equivalence-The ankle joint is a virtual revolute joint on the ground, and its axis of rotation can be arbitrarily placed without changing the mechanism mobility.

\subsection{The Equivalent RUSR Linkage}

For simplicity of kinematic and force analyses, ths $7 \mathrm{R}$ linkage is modelled as an equivalent RUSR linkage as shown in Fig. 2(b). In this simplified kinematic structure, the joints $R_{2}$ and $R_{3}$ are equivalent to a universal joint $U_{1}$, whereas the joints $R_{4}, R_{5}$ and $R_{6}$ are equivalent to a spherical joint $S_{1}$. Because the $7 R$ and the RUSR linkages are kinematically equivalent, their displacement and force relationships between the input and output joints will be identical. Therefore, the four-bar RSUR linkage will be employed for the analyses for the rehabilitation device in the follows.

\section{Displacement Analysis}

Since the displacement of the RUSR linkage is equivalent to the RSSR linkage, here we carry out the displacement analysis of the linkage by using the equations for the RSSR linkage, which are available in [5]. Fig. 3(a) shows the schematic of using the RSSR linkage for ankle rehabilitation while taking the ankle joint as a virtual revolute joint. To describe the rehabilitation motion of the RSSR linkage, two position parameters should be considered, i.e., the inclination angle $\varphi$ of the foot and the rotation angle $\gamma$ of the joint axis of the virtual ankle joint (see Fig. 3(b)). Also, $\theta_{1}$ and $\theta_{4}$ represent the rotation angles of the actuation motor and the ankle motion, respectively. 


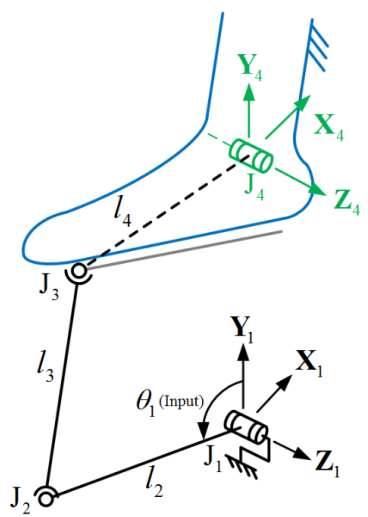

(a) Schematic of using the RSSR linkage

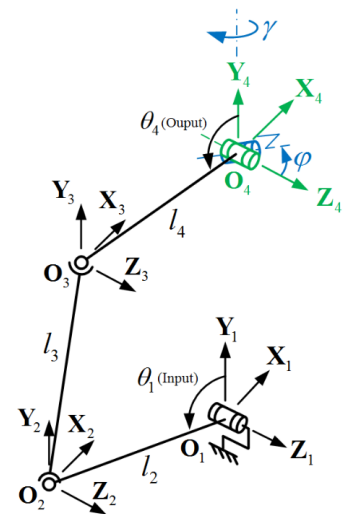

(b) Position parameters $\varphi$ and $\gamma$

Fig. 3. The equivalent RSSR linkage.

To understand the relationship between $\theta_{1}$ and $\theta_{4}$, four coordinate systems corresponding to the four joints $\mathrm{J}_{1}, \mathrm{~J}_{2}, \mathrm{~J}_{3}$, and $\mathrm{J}_{4}$ are defined as Fig. 3(b). The first coordinate system (with axis $x_{1}, y_{1}, z_{1}$ and origin $\mathbf{O}_{1}$ ) and fourth coordinate system (with axis $x_{4}, y_{4}, z_{4}$ and origin $\mathbf{O}_{4}$ ) are attached to the input and output joints, where axes $\mathbf{Z}_{1}$ and $\mathbf{Z}_{4}$ coincide with the rotation axes of $\mathbf{J}_{1}$ and $\mathbf{J}_{4}$. The variable direction of axis $Z_{4}$ implies the changeable ankle rotation axis. The second and third coordinate system, $\mathbf{J}_{2}$ and $\mathbf{J}_{3}$, are located on the planes defined by $\left(X_{1}, Y_{1}\right)$ and $\left(X_{4}, Y_{4}\right)$, respectively.

Thus, the relation between the input and output angles $\left(\theta_{1}\right.$ and $\left.\theta_{4}\right)$ can be obtained as follows [6]:

$$
\begin{gathered}
\theta_{4}=\tan ^{-1}\left(\frac{e}{d}\right) \pm \cos ^{-1}\left(\frac{f}{\sqrt{d^{2}+e^{2}}}\right)-\frac{\pi}{2} \\
d=2 l_{4} s_{11}\left(x_{14}-l_{2} \cos \theta_{1}\right)+2 l_{4} s_{21}\left(y_{14}-l_{2} \sin \theta_{1}\right)+2 l_{4} s_{31} z_{14} \\
e=2 l_{4} s_{12}\left(x_{14}-l_{2} \cos \theta_{1}\right)+2 l_{4} s_{22}\left(y_{14}-l_{2} \sin \theta_{1}\right)+2 l_{4} s_{32} z_{14} \\
f=l_{3}^{2}-l_{4}^{2}-l_{2}^{2}-x_{14}^{2}-y_{14}^{2}-z_{14}^{2}+2 l_{2} x_{14} \cos \theta_{1}+2 y_{14} l_{2} \sin \theta_{1}
\end{gathered}
$$

where the general homogeneous matrix of the relationship between $\mathbf{J}_{1}$ and $\mathbf{J}_{4}$ can be formulated by

$$
\mathbf{M}_{14}=\left[\begin{array}{cc}
\mathbf{R}_{14} & \left(\mathbf{O}_{4}-\mathbf{O}_{1}\right) \\
0 & 1
\end{array}\right]=\left[\begin{array}{cccc}
s_{11} & s_{12} & s_{13} & x_{14} \\
s_{21} & s_{22} & s_{23} & y_{14} \\
s_{31} & s_{32} & s_{33} & z_{14} \\
0 & 0 & 0 & 1
\end{array}\right]
$$


By referring to the general rehabilitation need, we adopt the linkage geometric parameters as listed in Table 1. Accordingly, the relationship between the input and output angles of the RSSR linkage can be obtained as Fig. 4. It shows that the output angle is nearly proportional to the input angle.

Table 1. The geometric parameters of the RSSR linkage for the ankle rehabilitation device.

\begin{tabular}{ccccc}
\hline$l_{2}$ & $l_{3}$ & $l_{4}$ & $\gamma$ & {$\left[x_{14}, y_{14}, z_{14}\right]^{\mathrm{T}}$} \\
\hline $150 \mathrm{~mm}$ & $70 \mathrm{~mm}$ & $160 \mathrm{~mm}$ & $0^{\circ}$ & {$[0,40,0]^{\mathrm{T}} \mathrm{mm}$} \\
\hline
\end{tabular}

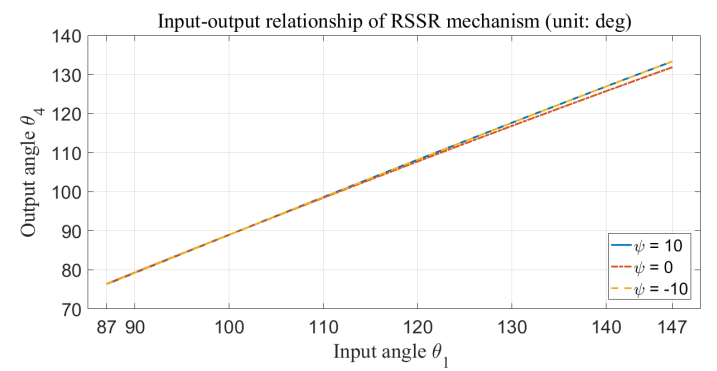

Fig. 4. The relationship between the input and output angles of the RSSR linkage for ankle rehabilitation. ( $\psi$ in degrees)

\section{$5 \quad$ Static Force Analysis}

For an ankle rehabilitation mechanism, knowing the load imposed on the ankle by the mechanism is essential. Based on the principle of static equilibrium, the static forces associated with every link in the linkage can be obtained. In this study, we assume that 1) the motor torque is $15 \mathrm{~N}-\mathrm{m}, 2$ ) the foot weight applied on the middle of link $l_{4}$ is $4.12 \mathrm{kgf}$, and 3) all the link masses are neglected. Thus, according to the dimension in Table 1, the variation of the reaction force at the ankle joint (i.e., the force that the mechanism exerts on the patient's ankle) is shown in Fig. 5. Besides, the variation of the output torque at the ankle joint (i.e., the torque that the mechanism applies on the patient's foot) is shown in Fig. 6.

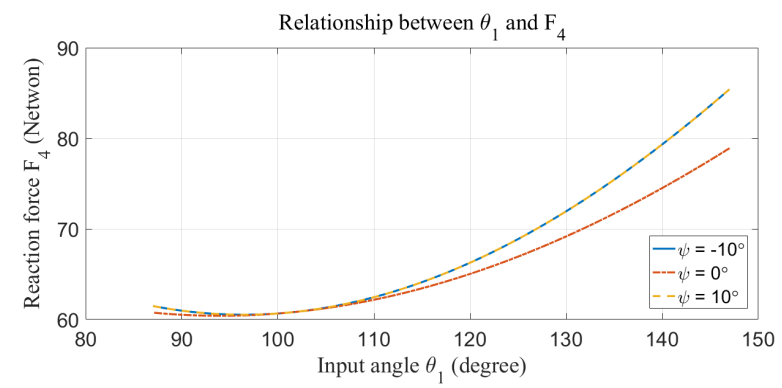

Fig. 5. The variation of the reaction force at the ankle joint. 


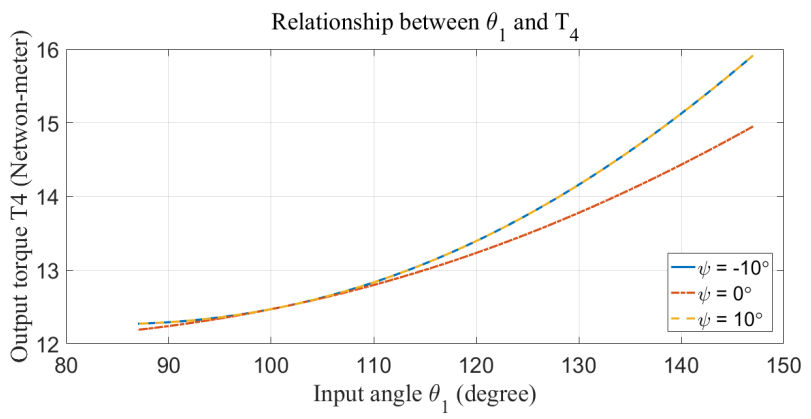

Fig. 6. The variation of the output torque at the ankle joint.

\section{Mechanical Implementation}

\subsection{Parallelogram Enhancement}

For improving the strength of the mechanical device, a parallelogram linkage is employed in the prototype. Fig. 7(a) shows the primary structure of the original 7R, while Fig. 7(b) illustrates the enhanced parallelogram design. Note again that the kinematics of the two linkages in Fig. 7(a) and (b) are equivalent, but the mechanical strength of the parallelogram-based design can be inherently augmented.

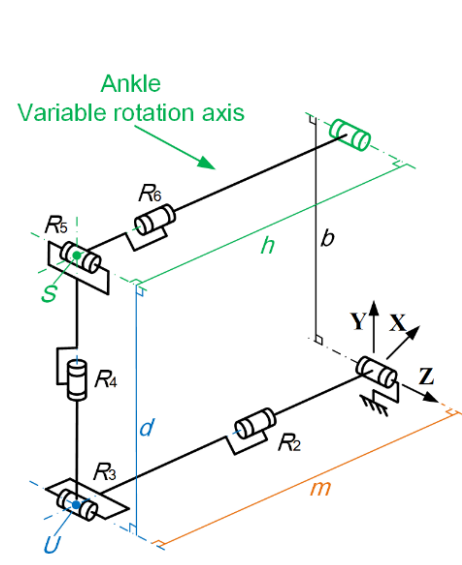

(a) The original $7 \mathrm{R}$ design

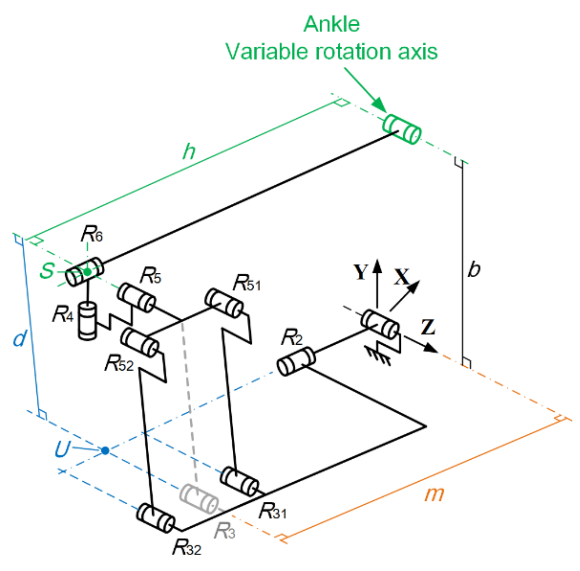

(b) The enhanced parallelogram design

Fig. 7. A parallelogram design for enhancing the strength of the $7 R$ linkage.

\subsection{Prototype}

A prototype of the proposed ankle rehabilitation device was built up as Fig. 8. In this prototype, particularly, a curve guide, namely Gonio Way [6], was used to mimic the rotational motion of joint $R_{6}$ in Fig. 7(b). The use of such a curve guide can effective- 
ly simplify the mechanical structure of the device. On the other hand, a set of forceregulating cables [7] was employed to support the virtual joint of the ankle. Fig. 9 shows the use of the device for ankle rehabilitation.

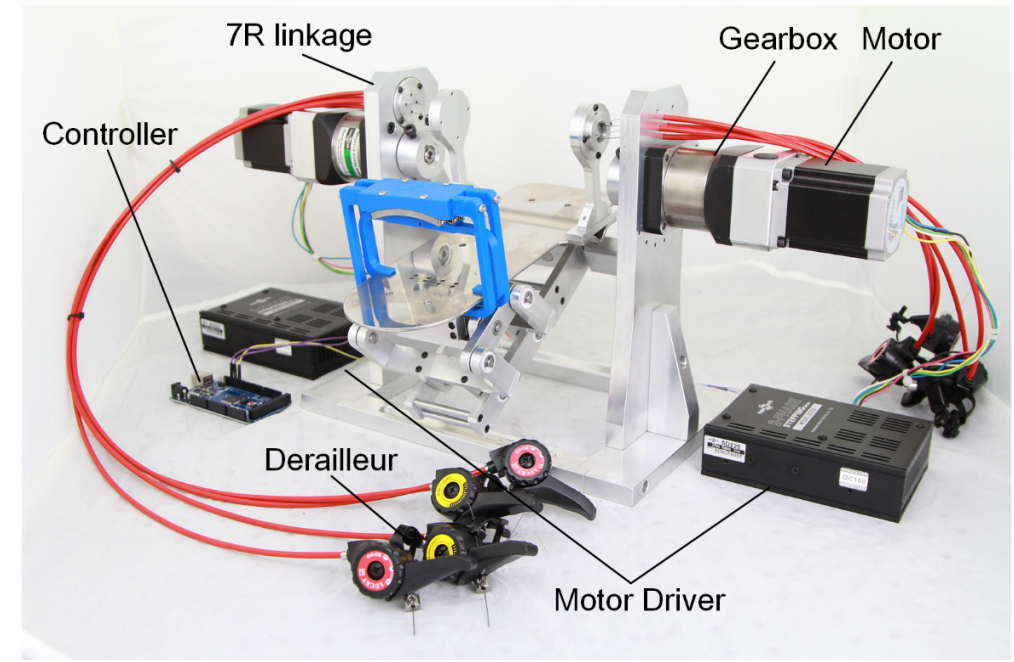

Fig. 8. Overview of the proposed rehabilitation device.

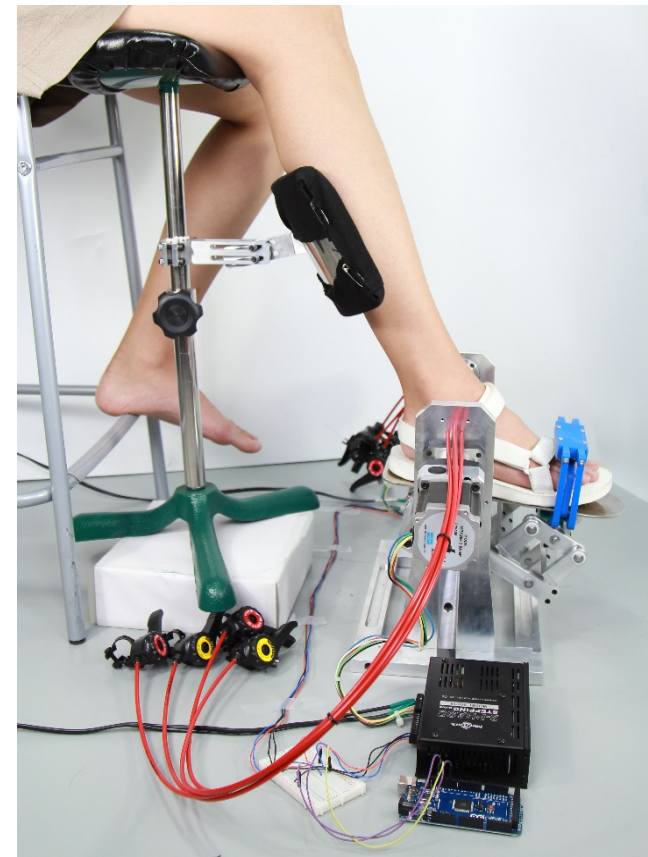

Fig. 9. Use of the proposed rehabilitation device. 


\section{Conclusions}

A 7R linkage and its equivalent kinematic and force analysis for ankle rehabilitation were presented in this paper. The proposed 7R design can lead the patient's ankle performing an arbitrary 3-D motion during rehabilitation exercise. The kinematic and static force analyses of the linkage were performed through an equivalent RSSR mechanism. Given by a defined dimension of the mechanism, the kinematic analysis shows that the input and output joint angles of the device are proportional. Besides, the force analysis can determine the reaction force at the ankle joint. A prototype was made for implementing the design idea. Future work is expected on the improvement of mechanical design with a more compact structure.

\section{References}

1. Alcocer, W., Vela, L., Blanco, A., Gonzalez, J., Oliver, M.: Major trends in the development of ankle rehabilitation devices. DYNA (Colombia), 79(176), 45-55 (2012).

2. Saglia, J.A., Tsagarakis, N.G., Dai, J.S., Caldwell, D.G.: A high-performance redundantly actuated parallel mechanism for ankle rehabilitation. International Journal of Robotics Research, 28(9), 1216-1227 (2009).

3. Girone, M.J., Burdea, G.C., Bouzit, M.: 'Rutgers ankle' orthopedic rehabilitation interface. ASME International Mechanical Engineering Congress and Exposition, Nashville, TN, USA (1999).

4. Matsuura, D., Koga, T., Ishida, S., Takeda, Y.: Kinetostatic design of ankle rehabilitation mechanism capable of adapting to changes in joint axis. Journal of Robotics and Mechatronics, 25(6), 1029-1037 (2013).

5. Mazzotti, C., Troncossi, M., Parenti-Castelli, V.: Dimensional synthesis of the optimal RSSR mechanism for a set of variable design parameters. Meccanica, 52(10), 2439-2447 (2017).

6. Nippon Bearing Co. Ltd., http://www.nb-linear.co.jp/

7. Hsieh, C.-C.: A passive ankle rehabilitation device based on a novel 7R spatial linkage. Master Thesis, Department of Mechanical Engineering, National Taiwan University of Science and Technology, Taipei, Taiwan (2018). 